\title{
Prevalencia de obesidad en una cohorte de pacientes con artritis psoriásica y factores asociados
}

\author{
C.A. Zaffarana, E.E. Schneeberger, J. Gallino Yanzi, O. Cerda, M. Landi, G.Citera \\ Instituto de Rehabilitación Psicofísica, CABA, Argentina
}

\begin{abstract}
Resumen
Se ha observado elevada prevalencia de obesidad en pacientes con Artritis Psoriásica (APs). También, ha sido descripta asociación con una mayor severidad de psoriasis cutánea, pero su relación con la severidad de la APs ha sido menos estudiada.

Objetivos: Evaluar la prevalencia de obesidad en una cohorte de pacientes con APs y su asociación con características sociodemográficas y clínicas de la enfermedad.
\end{abstract}

Material y métodos: Se estudiaron pacientes consecutivos $\geq 18$ años de edad con diagnóstico de APs según criterios CASPAR de la cohorte RAPSODIA. Se recolectaron datos sociodemográficos, tiempo de evolución, forma de inicio, de evolución de la APs, comorbilidades y tratamiento. Se evaluó la actividad de la enfermedad mediante el recuento de 66/68 articulaciones tumefactas y dolorosas, respectivamente. Índices compuestos DAS28, DAPSA, cuestionario BASDAl y criterios de MDA. Capacidad funcional (HAQ-A, BASFI), calidad de vida (DLQI, PSAQoL), compromiso cutáneo (PASI), ungueal (PNSS), entesis (MASES) y la presencia de dactilitis. Se utilizó escala visual análoga (EVA) para graduar severidad de psoriasis cutánea, dolor y evaluación global de la actividad de la enfermedad según médico (EGEm) y paciente (EGEp). Se realizó examen físico con medición de talla $(\mathrm{m})$, peso $(\mathrm{kg})$ y tensión arterial $(\mathrm{mm} \mathrm{Hg})$. Se calculó el Índice de Masa Corporal (IMC)=peso/talla ${ }^{2}$. Se clasificó a los pacientes según la Clasificación Internacional del estado nutricional de la OMS en: Normal (IMC 18,5-24,9), Sobrepeso (IMC $25-29,9$ ) y Obesidad (IMC $\geq 30$ ). Obesidad se clasificó en grado I 0 leve (IMC 30-34,9), grado II o moderada (IMC 35-39,9) y grado III 0 severa (IMC $\geq 40$ ). Análisis Estadístico: Estadística descriptiva. T test y ANOVA. Chi ${ }^{2}$ y test exacto de Fisher. Modelo de regresión logística múltiple (variable dependiente: obesidad).

Resultados: Se incluyeron 110 pacientes con APs. $56(50,9 \%)$ eran hombres. La mediana de IMC fue 28,38 (RIC: 15,5-32,2). 21 $(19,1 \%)$ tenían IMC normal, 48 (43,6\%) sobrepeso, 41 (37,3\%) obesidad. De los pacientes obesos, $33(80,5 \%)$ tenían obesidad leve, $6(14,6 \%)$ moderada y $2(4,9 \%)$ severa. Los pacientes obesos tenían peor capacidad funcional BASFl: $4,4 \pm 2,8$ vs. $2,7 \pm 2,5$,

\section{Abstract}

A high prevalence of obesity has been observed in patients with Psoriatic Arthritis (PsA). Also, an association with a greater severity of cutaneous psoriasis has been described, but its relationship with the severity of PsA has been less studied.

Objectives: To evaluate the prevalence of obesity in a cohort of patients with PsA and their association with sociodemographic and clinical characteristics of the disease.

Material and methods: We studied consecutive patients $\geq 18$ years of age with a diagnosis of PSA according to CASPAR criteria of the RAPSODIA cohort. Sociodemographic data, time of evolution, form of onset, evolution of PsA, comorbidities and treatment were collected. Disease activity was assessed by counting 66/68 swollen and painful joints, respectively. DAS28 composite indices, DAPSA, BASDAl questionnaire and MDA criteria. Functional capacity (HAQ-A, BASFI), quality of life (DLQI, PsAQoL), cutaneous involvement (PASI), nail (PNSS), entesis (MASES) and presence of dactylitis. An analogous visual scale (AVS) was used to grade cutaneous psoriasis severity, pain and overall assessment of disease activity according to physician (EGEm) and patient (EGEp). Physical examination was performed with measurement of height $(\mathrm{m})$, weight $(\mathrm{kg})$ and blood pressure $(\mathrm{mm} \mathrm{Hg})$. The Body Mass Index $(\mathrm{BMI})=$ weight $_{\text {/height }}{ }^{2}$ was calculated. Patients were classified according to the WHO International Classification of Nutrition Status in: Normal (BMI 18.524.9), Overweight (BMI 25-29.9) and Obesity (BMI $\geq 30$ ). Obesity was classified as grade I or mild (BMI 30-34.9), grade II or moderate (BMI 35-39.9) and grade III or severe (BMI $\geq 40$ ). Statistical Analysis: Descriptive Statistics. T test and ANOVA. Chi ${ }^{2}$ and Fisher's exact test. Multiple logistic regression model (dependent variable: obesity). Results: We included 110 patients with PsA. 56 (50.9\%) were men. The median BMI was 28.38 (BER: 15.5-32.2). 21 (19.1\%) had normal BMI, 48 (43.6\%) were overweight, 41 (37.3\%) were obese. Of the obese patients, $33(80.5 \%)$ had mild obesity, 6 (14.6\%) moderate and $2(4.9 \%)$ severe obesity. Obese patients had worse BASFI functional capacity: $4.4 \pm 2.8$ vs. $2.7 \pm 2.5, p=0.03$ ) and greater pain (6.7 \pm 7.6 vs. $4.6 \pm 2.4, p=0.048)$. No patient with normal weight 
$p=0,03)$ y mayor dolor $(6,7 \pm 7,6$ vs. $4,6 \pm 2,4, p=0,048)$. Ningún paciente con normopeso tenía DBT tipo II, vs. 16 pacientes $(18 \%)$ del grupo sobrepeso y obesidad $(p=0,03)$. La media de PASI era mayor en el grupo con obesidad y sobrepeso pero esta diferencia perdió significancia estadística al ajustar por tiempo de evolución. En el análisis de regresión logística múltiple, luego de ajustar por diferentes variables (edad, sexo, capacidad funcional, tiempo de evolución y dolor), el tratamiento con esteroides permaneció asociado a la presencia de sobrepeso y obesidad (OR: 3,93, IC 95\%: 1,29-11,9, $p=0,016)$.

Conclusiones: Más del $80 \%$ de los pacientes de nuestra cohorte presentaron un IMC anormal. La presencia de obesidad se asoció a peor capacidad funcional por BASFI y a mayor dolor, y tener un IMC $\geq 25$ se asoció a la presencia de DBT II. El tratamiento con esteroides fue la única variable independientemente asociada a sobrepeso y a obesidad.

Palabras clave: obesidad, prevalencia, artritis psoriásica. had type II DBT, vs. 16 patients (18\%) were overweight and obese $(p=0.03)$. Mean PASI was higher in the obese and overweight group but this difference lost statistical significance when adjusted for evolution time. In the multiple logistic regression analysis, after adjusting for different variables (age, sex, functional capacity, time of evolution and pain), steroid treatment remained associated with the presence of overweight and obesity (OR: $3.93,95 \% \mathrm{Cl}$ : 1.29 11.9, $\mathrm{p}=0.016$ ).

Conclusions: More than $80 \%$ of our cohort patients had an abnormal BMI. The presence of obesity was associated with worse functional capacity by BASFI and greater pain, and a BMI $\geq 25$ was associated with the presence of DBT II. Steroid treatment was the only variable independently associated with overweight and obesity.

Key words: obesity, prevalence, psoriatic arthritis.

\section{Introducción}

La Artritis Psoriásica (APs) es una artropatía inflamatoria que presenta compromiso articular tanto periférico como axial, y se asocia con psoriasis cutánea y/o ungueal. Afecta entre un 7-42\% de los pacientes con Psoriasis cutánea ${ }^{1}$. La obesidad constituye un factor de riesgo para enfermedad cardiovascular, es uno de los disturbios que integran el síndrome metabólico y es considerada uno de los principales factores de riesgo de muerte a nivel mundial. La prevalencia de obesidad es considerablemente alta en pacientes con psoriasis y $\mathrm{APs}^{2}$. Estudios prospectivos observaron que la obesidad sería un factor predictor de desarrollo para ambas enfermedades y tendría un impacto negativo sobre el pronóstico de las mismas ${ }^{3-5}$.

La obesidad provoca un estado proinflamatorio con liberación de citoquinas como el TNF- $\alpha$ por los adipocitos. Además el tejido adiposo produce una cantidad de moléculas con actividad metabólica que comparten características funcionales y estructurales con las citoquinas, denominadas adipoquinas. Los pacientes obesos con Psoriasis tienen mayor probabilidad de desarrollar enfermedad cutánea diseminada y presentan menor respuesta a los tratamientos, mientras que la disminución de peso se asoció con mejoría de la enfermedad ${ }^{6-10}$.

Si bien la relación de la obesidad con la APs ha sido objeto de estudio a nivel mundial, no contamos con datos de nuestro país, por lo que nos proponemos como objetivos evaluar la prevalencia de obesidad en una cohorte de pacientes con APs y su asociación con características sociodemográficas y clínicas de la enfermedad.

\section{Material y métodos}

Se realizó un estudio de corte transversal en pacientes consecutivos $\geq 18$ años de edad con diagnóstico de APs según criterios CASPAR ${ }^{11}$. Se recolectaron datos sociodemográficos (edad, sexo, estado civil, ocupación), 
tiempo de evolución, forma de inicio y de evolución de la APs, presencia de comorbilidades y laboratorio incluyendo reactantes de fase aguda (ERS -mm/h- y PCR -mg\%-) y perfil lipídico (colesterol total, HDL, LDL, triglicéridos -mg/\%-). Se recabaron datos del tratamiento por medio de una entrevista directa con el paciente complementando con la historia clínica. Se evaluó actividad de la enfermedad mediante el recuento de 66/68 articulaciones tumefactas y dolorosas, respectivamente. Además se calcularon los índices compuestos DAS28 (Disease Activity Score in 28 joints) ${ }^{12}$ y DAPSA (Disease Activity in Psoriatic Arthritis) ${ }^{13}$, el autocuestionario BASDAI (Bath Ankylosing Spondylitis Disease Activity Index $)^{14,15}$, y se consideraron los criterios de MDA (Minimal Disease Activity) ${ }^{16}$. La capacidad funcional se determinó por el autocuestionario HAQ (Health Assessment Questionnaire disability index $)^{17}$, BASFI (Bath Ankylosing Spondylitis Metrology Index) ${ }^{18}$. Se evaluó calidad de vida por DLQI (Dermatology Life Quality Index) ${ }^{19}$ y PsAQoL (Psoriatic Arthritis Quality of Life $)^{20}$. Todos los autocuestionarios utilizados fueron validados al español en nuestro centro en Argentina. El compromiso cutáneo se evaluó por PASI (Psoriasis Area and Severity Index $)^{21}$, los sitios de entesis por MASES (Maastricht Ankylosing Spondylitis Enthesis Score) ${ }^{22}$ y mediante examen físico la presencia de dactilitis. Todo el examen físico, incluyendo la evaluación del compromiso cutáneo, fue realizado por el médico reumatólogo que realizó la entrevista con el paciente. Se utilizó escala visual análoga (EVA) para graduar severidad de psoriasis cutánea, dolor y evaluación global de la actividad de enfermedad por el paciente (EGEp) y evaluación global de la actividad de enfermedad por el médico (EGEm). Las formas clínicas de APs se clasificaron en los cinco grupos clásicos descriptos por Moll y Wright ${ }^{23}$, y según la afectación del esqueleto axial se los clasificó en compromiso periférico puro, axial puro o mixto. La afectación del esqueleto axial se definió según la presencia de dolor lumbar inflamatorio, limitación funcional de la movilidad del esqueleto axial, y evidencia por estudios por imagen (sindesmofitos, sacroileítis radiológica o por Resonancia Magnética Nuclear) ${ }^{24}$. Se realizó examen físico con medición de talla $(\mathrm{cm})$, peso $(\mathrm{kg})$ y tensión arterial. Se calculó el Índice de Masa Corporal $(\mathrm{IMC})=$ peso $/ \mathrm{talla}^{2}$. Se clasificó a los pacientes según la Clasificación Internacional del estado nutricional de la OMS en: Normal (IMC 18,5-24,9), Sobrepeso (IMC 25-
29,9) y Obesidad (IMC 230 ). Obesidad se clasificó en grado I o leve (IMC 30-34,9), grado II o moderada (IMC 35-39,9) y grado III o severa (IMC $\geq 40$ ).

Para el análisis estadístico se realizó estadística descriptiva. Las variables categóricas se expresaron en frecuencia y porcentaje y las continuas en medianas y RIC (rango intercuartilo) o en medias y DS (desvío standard). Las variables categóricas se compararon por test de $\mathrm{Chi}^{2}$ y test exacto de Fisher y las continuas por test de Student y ANOVA. Para evaluar las variables asociadas independientemente con la presencia de obesidad se utilizó un modelo de regresión logística múltiple.

\section{Resultados}

Se incluyeron 110 pacientes con APs pertenecientes a la cohorte RAPSODIA (ㄹegistro de Artritis PSOriásica Del

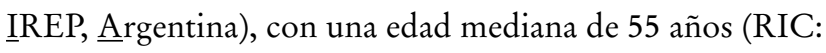
44,7-63,2), 56 pacientes $(50,9 \%)$ eran varones. El tiempo mediano de evolución de la APs fue 10 años (RIC: 6-17) y de la Psoriasis fue 24 años (RIC: 15,5-33,9). 55 pacientes (50\%) tenían compromiso periférico, $2(1,8 \%)$ axial puro y $53(48,2 \%)$ compromiso mixto. 19 pacientes $(17,3 \%)$ cumplían criterios de MDA (Tabla 1).

El IMC mediano fue 28,38 (RIC: 25,6-32,2). 21 pacientes $(19,1 \%)$ tenían IMC normal, 48 (43,6\%) sobrepeso, 41 (37,3\%) obesidad. De los pacientes obesos, $33(80,5 \%)$ tenían obesidad leve, 6 (14,6\%) moderada y 2 $(4,9 \%)$ severa.

Los pacientes obesos presentaron significativamente peor capacidad funcional que los pacientes con peso normal $(4,4 \pm 2,8$ vs. $2,7 \pm 2,5, \mathrm{p}=0,03)$ y mayor dolor (VAS $6,7 \pm 7,6$ vs. $4,6 \pm 2,4, p=0,048)$. No hubo diferencias según sexo, edad, comorbilidades, tiempo de evolución de la APs y la Psoriasis, forma de inicio y de evolución de la APs, variables del laboratorio, ni otras medidas de evaluación de la enfermedad (Tabla 2).

En relación al tratamiento, se observó que un número significativamente mayor de pacientes con sobrepeso y obesidad había recibido terapia con glucocorticoides, 9 $(13 \%)$ de los pacientes con peso normal, 37 (50\%) con sobrepeso y $28(37 \%)$ con obesidad, $(p=0,02)$. Sin embargo, al analizar solamente aquellos con tratamiento esteroideo al momento del estudio, no se observaron diferencias según el IMC (Tabla 2). 
Al comparar los pacientes con peso normal vs. aquellos con obesidad y sobrepeso, ningún paciente con normopeso tenía DBT tipo II versus 16 pacientes con sobrepeso y obesos $(\mathrm{p}=0,03)$. También el PASI fue significativamente superior en pacientes obesos y con sobrepeso vs. pacientes con peso normal $(2,8 \pm 2,9$ vs. $1,6 \pm 1,7, p=0,02)$. Sin embargo, esta diferencia perdió significancia estadística al ajustar por tiempo de evolución.

Por último, en el análisis de regresión logística múltiple, tomando como variable dependiente la presencia de sobrepeso y obesidad, luego de ajustar por diferentes variables (edad, sexo, capacidad funcional, tiempo de evolución y dolor), el tratamiento con esteroides permaneció asociado a la presencia de sobrepeso y obesidad. (OR: 3,93, IC 95: 1,29-11,9, p=0,016) (Tabla 3).

\section{Discusión}

En este estudio observamos que $81 \%$ de los pacientes con APs presentaron sobrepeso u obesidad. Solamente el 19\% de los pacientes presentaban un IMC normal. La presencia de DBT II fue significativamente superior en pacientes con sobrepeso y obesos.

La prevalencia de obesidad en nuestra cohorte de pacientes fue $37,3 \%$, y de sobrepeso $43,6 \%$. La prevalencia de obesidad y sobrepeso en APs fue superior al observado en la población general de nuestro país, de acuerdo a los datos publicados por la Encuesta Nacional de Factores de Riesgo para enfermedades no transmisibles (ENFR) $3^{\circ}$ edición, en los que las prevalencias de sobrepeso y obesidad en Argentina son 37,1\% y 20,8\%, respectivamente ${ }^{25}$. En la ENFR se informó que tanto el sobrepeso como la obesidad son más frecuentes en hombres y que el menor nivel educativo se asocia a mayor prevalencia de obesidad y sobrepeso. En nuestra cohorte, la prevalencia de sobrepeso y obesidad fue mayor numéricamente en hombres que en mujeres. Sin embargo, no encontramos diferencias estadísticamente significativas en cuanto a sexo, edad, nivel educativo ni tiempo de evolución de la enfermedad.

Los resultados de este trabajo son concordantes con los publicados por otros autores, quienes también hallaron una mayor prevalencia de obesidad en APs que en la población general. En el estudio realizado por Bhole y cols., se reportó que pacientes con APs tenían mayor IMC que pacientes con psoriasis cutánea, artritis reumatoidea y la población general. En este estudio, la proporción de obesos en APs fue del $37 \%$ versus $18 \%$ en la población de Canadá26.

Al evaluar la presencia de comorbilidades, hallamos una mayor frecuencia de DBT II en los pacientes con sobrepeso y obesidad en comparación a los pacientes con peso normal. Este hallazgo es de esperar ya que la DBT tipo II es más frecuentemente observada en pacientes obesos hiperinsulinémicos y con síndrome metabólico ${ }^{27}$. En nuestra cohorte, los pacientes con peso normal tuvieron menor severidad cutánea que aquellos pacientes con sobrepeso y obesidad. Sin embargo, al ajustar por tiempo de evolución, la diferencia en PASI perdió significancia estadística. El diseño de este trabajo no nos permite elaborar una posible explicación.

En nuestro estudio observamos que un menor porcentaje de pacientes con IMC en valores de sobrepeso y obesidad cumplieron criterios de MDA, y además estos pacientes presentaban mayor recuento de articulaciones inflamadas. Sin embargo, esto no fue estadísticamente significativo. No podemos descartar un sesgo por indicación en este caso, ya que un mayor porcentaje de obesos recibían tratamiento con Drogas Modificadoras de Enfermedad (DME) y agentes biológicos.

Observamosmayorfrecuenciadeusodeglucocorticoides en el pasado en pacientes obesos y con sobrepeso. Debido a que éste es un estudio retrospectivo en el que la recolección de datos se realizó en forma transversal, no se pudo calcular la dosis acumulada de esteroides, lo cual es una limitación para interpretar estos resultados.

Algunos estudios evaluaron la relación entre la obesidad y la severidad de la artritis en APs. Di Minno y cols. reportaron que un mayor IMC predice menor respuesta a la terapia anti-TNF $\alpha$ en pacientes seguidos por 24 meses. También, se observó que el descenso de peso se asoció a mayor respuesta a estos agentes ${ }^{28,29}$. Un estudio que evaluó la probabilidad de alcanzar MDA en pacientes con APs categorizados según Índice de Masa Corporal (IMC), halló que los pacientes con mayores valores de IMC tenían menor probabilidad de alcanzar MDA sostenido, y esta asociación fue independiente del uso de DME y de biológicos. Uno de los nexos entre obesidad e inflamación son las adipoquinas. Entre estas se encuentran la adiponectina, que es un regulador de la sensibilidad a la insulina, y la leptina, que es un reflejo de los niveles de masa 


\begin{tabular}{|c|c|}
\hline Variable & $n=110$ \\
\hline Sexo masculino $\mathrm{n}(\%)$ & $56(50,9)$ \\
\hline Edad $m(\mathrm{RIC})$ & $55(44,7-63,2)$ \\
\hline Tiempo de evolución APs años $m$ (RIC) & $10(6-17)$ \\
\hline Tiempo de evolución psoriasis años $m(\mathrm{RIC})$ & $24(15,5-33,9)$ \\
\hline IMC $m(\mathrm{RIC})$ & $28,4(15,5-32,2)$ \\
\hline Compromiso periférico puro $\mathrm{n}(\%)$ & $55(50,9)$ \\
\hline Axial puro $\mathrm{n}(\%)$ & $2(1,9)$ \\
\hline Mixto $\mathrm{n}(\%)$ & $53(48,2)$ \\
\hline
\end{tabular}

APs: Artritis Psoriásica. IMC: Índice de Masa Corporal. RIC: Rango Intercuartílico.

Tabla 1. Características sociodemográficas y clínicas en 110 pacientes con APs.

\begin{tabular}{|l|c|c|c|c|c|}
\hline Variable & B & p & OR & \multicolumn{2}{|c|}{ IC 95\% } \\
\cline { 5 - 7 } & & & & Inferior & Superior \\
\hline Sexo & 0,26 & 0,64 & 1,30 & 0,42 & 4,02 \\
\hline Edad & 0,03 & 0,14 & 1,03 & 0,98 & 1,08 \\
\hline Tiempo de evolución APS & $-0,03$ & 0,46 & 0,97 & 0,90 & 1,04 \\
\hline Dolor (EVG) & $-0,02$ & 0,72 & 0,97 & 0,87 & 1,10 \\
\hline BASFI & 0,15 & 0,21 & 1,16 & 0,91 & 1,49 \\
\hline Tratamiento esteroideo & $\mathbf{1 , 3 7}$ & $\mathbf{0 , 0 1 6}$ & $\mathbf{3 , 9 3}$ & $\mathbf{1 , 2 9}$ & $\mathbf{1 1 , 9 6}$ \\
\hline PASI & 0,24 & 0,08 & 1,27 & 0,96 & 1,69 \\
\hline
\end{tabular}

Variable dependiente: sobrepeso y obesidad.

Tabla 3. Análisis de regresión logística múltiple. Variable dependiente: sobrepeso y obesidad.

\begin{tabular}{|c|c|c|c|c|c|}
\hline \multicolumn{2}{|l|}{ Variable } & \multicolumn{2}{|c|}{ Peso normal Sobrepeso } & \multirow{2}{*}{$\begin{array}{c}\text { Obesidad } \\
n=41\end{array}$} & \multirow{2}{*}{\begin{tabular}{|c|}
$\mathbf{p}$ \\
NS \\
\end{tabular}} \\
\hline & & $\mathrm{n}=21$ & $\mathrm{n}=48$ & & \\
\hline \multirow{2}{*}{$\begin{array}{l}\text { Características } \\
\text { sociodemo- } \\
\text { gráficas }\end{array}$} & $\begin{array}{l}\text { Sexo femenino } \\
\mathrm{n}(\%)\end{array}$ & $13(61,9)$ & $24(50)$ & $17(41,5)$ & NS \\
\hline & Edad años $\mathrm{X} \pm \mathrm{DS}$ & $50,2(11,0)$ & $52,8(13,7)$ & $56,8(12,3)$ & NS \\
\hline \multirow[t]{6}{*}{ Autocuestionarios } & BASFI $\mathrm{X} \pm \mathrm{DS}$ & $2,7(2,5)^{*}$ & $3,3(2,7)$ & $4,4(2,8)^{*}$ & $0,03^{*}$ \\
\hline & BASDAI $X \pm D S$ & $3,8(2,5)$ & $3,9(2,9)$ & $4,9(2,8)$ & NS \\
\hline & AsQoL $X_{ \pm} D S$ & $3,7(5,3)$ & $4,1(5,3)$ & $5,1(6,2)$ & NS \\
\hline & PsAQoL X \pm DS & $4,7(5,3)$ & $6,4(6,1)$ & $8,5(6,5)$ & 0,05 \\
\hline & HAQ $X_{ \pm} \mathrm{DS}$ & $0,63(0,6)$ & $0,71(0,6)$ & $0,96(0,6)$ & NS \\
\hline & Dolor $X_{ \pm} \mathrm{DS}$ & $4,6(2,4)$ & $4,3(2,9)$ & $6,7(7,6)^{*}$ & $0,048^{\star}$ \\
\hline \multirow[t]{3}{*}{ Examen físico } & $\begin{array}{l}\text { Rto Articulaciones } \\
\text { tumefactas } X \pm D S\end{array}$ & $3,4(3,1)$ & $3,7(2,7)$ & $4,6(6,6)$ & NS \\
\hline & $\begin{array}{l}\text { Rto Articulaciones } \\
\text { dolorosas } \mathrm{X} \pm \mathrm{DS}\end{array}$ & $4,4(3,7)$ & $4,4(3,5)$ & $4,5(3,5)$ & NS \\
\hline & MASES $X \pm D S$ & $1,5(2,9)$ & $1,3(1,9)$ & $1,5(2,3)$ & NS \\
\hline \multirow{3}{*}{$\begin{array}{l}\text { Compromiso } \\
\text { cutáneo }\end{array}$} & PNSS $X \pm D S$ & $6,5(7,5)$ & $8,4(7,6)$ & $6,7(7,9)$ & NS \\
\hline & PASI $X \pm D S$ & $1,5(1,7)$ & $3,1(2,8)$ & $2,5(2,9)$ & NS \\
\hline & $P G A X \pm D S$ & $1,2(1,3)$ & $2,2(1,5)$ & $1,9(1,7)$ & 0,05 \\
\hline \multirow{2}{*}{$\begin{array}{l}\text { Índices } \\
\text { compuestos }\end{array}$} & MDA n (\%) & $5(23,8)$ & $10(20,8)$ & $4(9,75)$ & NS \\
\hline & CPDAI $\mathrm{X} \pm \mathrm{DS}$ & $5,8(3,6)$ & $6,6(3,9)$ & $8(3,5)$ & NS \\
\hline \multirow[t]{5}{*}{ Tratamiento } & $\begin{array}{l}\text { Glucocorticoides } \\
\text { (pasado) n (\%) }\end{array}$ & $9(42,8)$ & $37(77,1)^{\star}$ & $28(68,3)^{\star}$ & $0,02^{*}$ \\
\hline & $\begin{array}{l}\text { Glucocorticoides } \\
\text { (presente) n (\%) }\end{array}$ & $5(23,8)$ & $11(22,9)$ & $9(21,9)$ & NS \\
\hline & AINES n (\%) & $18(85,7)$ & $34(70,8)$ & $27(65,8)$ & NS \\
\hline & DME n (\%) & $14(66,6)$ & $32(66,6)$ & $35(85,3)$ & NS \\
\hline & Anti-TNF n (\%) & $3(14,3)$ & $4(8,3)$ & $10(24,4)$ & NS \\
\hline
\end{tabular}

DME: Drogas Modificadoras de Enfermedad.

Tabla 2. Características clínicas en 110 pacientes con APS según IMC.

explicaciones, entre ellas que participarían fenómenos inflamatorios relacionados a la producción de citoquinas y a trastornos musculo-esqueléticos causados por los efectos mecánicos del exceso de peso sobre las articulaciones ${ }^{34}$.

Concluimos, que más del 80\% de los pacientes con APs en Argentina presentan sobrepeso y obesidad. Los pacientes obesos con APs tienen peor capacidad funcional y mayor dolor. La DBT II es una comorbilidad frecuentemente observada en pacientes con APs con IMC anormal.

\section{Bibliografía}

1. Gladman DD, Antoni C, Mease P, Clegg DO, Nash 
P. Psoriatic arthritis: epidemiology, clinical features, course, and outcome. Ann Rheum Dis 2005;64(Suppl. 2):ii14-7.

2. Han C, Robinson DW Jr, Hackett MV, Paramore LC, Fraeman KH, Bala MV. Cardiovascular disease and risk factors in patients with rheumatoid arthritis, psoriatic arthritis, and ankylosing spondylitis. J Rheumatol 2006;33(11):2167-72.

3. Jamnitski A, Symmons D, Peters MJ, Sattar N, McInnes I, Nurmohamed MT. Cardiovascular comorbidities in patients with psoriatic arthritis: a systematic review. Ann Rheum Dis 2013;72(3):211-6.

4. Mok CC, Ko GT, Ho LY, Yu KL, Chan PT, To $\mathrm{CH}$. Prevalence of atherosclerotic risk factors and the metabolic syndrome in patients with chronic inflammatory arthritis. Arthritis Care Res (Hoboken) 2011;63(2):195-202.

5. Kumar S, Han J, Li T Qureshi AA. Obesity, waist circumference, weight change and the risk of psoriasis in US women. J Eur Acad Dermatol Venereol 2013;27(10)1293-8.

6. Love TJ, Zhu Y, Zhang Y, Wall-Burns L, Ogdie A, Gelfand JM, et al. Obesity and the risk of psoriatic arthritis: a population-based study. Ann Rheum Dis 2012;71(8):1273-7.

7. Armstrong AW, Harskamp CT, Armstrong EJ. The association between psoriasis and obesity: a systematic review and meta-analysis of observational studies. Nutr Diabetes 2012;2:e54

8. Carrascosa JM, Vilavella M, Garcia-Doval I, Carretero G, Vanaclocha F, Daudén E, et al. Body mass index in patients with moderate-to-severe psoriasis in Spain and its impact as an independent risk factor for therapy withdrawal: results of the Biobadaderm Registry. J Eur Acad Dermatol Venereol 2014;28(7):907-14.

9. Jensen P, Zachariae C, Christensen R, Geiker NR, Schaadt BK, Stender S, et al. Effect of weight loss on the severity of psoriasis: a randomized clinical study. JAMA Dermatol 2013;149(7):795-801.

10. Zhang C, Zhu KJ, Zheng HF, Cui Y, Zhou FS, Chen YL, et al. The effect of overweight and obesity on psoriasis patients in Chinese Han population: a hospital-based study. J Eur Acad Dermatol Venereol 2011;25(1):87-91. 11. Taylor W, Gladman D, Helliwell P, Marchesoni A, Mease P, Mielants H, and the CASPAR Study
Group. Classification criteria for psoriatic arthritis: development of new criteria from a large international study. Arthritis Rheum 2006;54:2665-73.

12. Smolen JS, Breedveld FC, Eberl G, Jones I, Leeming M, Wylie GL, et al. Validity and reliability of the twentyeight-joint count for the assessment of rheumatoid arthritis activity. Arthritis Rheum 1995;38(1):38-43.

13. Schoels M, Aletaha D, Funovits J, Kavanaugh A, Baker $\mathrm{D}$, Smolen JS. Application of the DAREA/DAPSA score for assessment of disease activity in psoriatic arthritis. Ann Rheum Dis 2010;69(8):1441-7

14. Citera G, Maldonado Cocco J, Moroldo M, BurgosVargas R, Anaya J, López I, et al. Validación de la versión en español de los cuestionarios de capacidad funcional (BASFI) y actividad de la enfermedad (BASDAI) en pacientes con Espondilitis Anquilosante en cuatro países latinoamericanos. Rev Arg Reumatol 1999;10(1):25.

15. Garret S, Jenkinson T, KennedyLG, Whitelock H, Gaisfold P, Calin A. A new approach to defining disease status in ankylosing spondylitis: the Bath Anlylosing Spondylitis DiseaseActivity Index. J Rheumatol 1994;21(12):2286-91.

16. Coates LC, Helliwell PS. Validation of minimal disease activity criteria for psoriatic arthritis using interventional trial data. Arthritis Care Res (Hoboken) 2010;62(7):965-9.

17. Citera G, Arriola MS, Maldonado Cocco JA, Rosemffet MA, Goñi MA, Spindler A, et al. Validation and cross cultural adaptation of an Argentine Spanish version of the health assessment questionnaire disability index. J Clin Rheumatol 2004;10(3):110-5.

18. Calin A, Garret S, Whitelock H, Kennedy LG, O’Hea J, Mallorie P, et al. A new approach to defining functional ability in ankylosing spondylitis: the development of the Bath Anlylosing Spondylitis Functional Index. J Rheumatol 1994;21(12):2281-5.

19. Finlay AY, Khan GK. Dermatology Life Quality Index (DLQI): a simple practical measure for routine clinical use. Clin Exp Dermatol 1994;19(3):210-6.

20. McKenna SP, Doward LC, Whalley D, Tennant A, Emery P, Veale DJ. Development of the PsAQoL: a quality of life instrument specific to psoriatic arthritis. Ann Rheum Dis 2004;63(2):162-9.

21. Fredriksson T, Pettersson U. Severe psoriasis: 
oral therapy with a new retinoid. Dermatologica 1978;157(4):238-44.

22. Heuft-Dorenbosch L, Spoorenberg A, van Tubergen A, Landewe $\mathrm{R}$, van ver Tempel $\mathrm{H}$, Mielants $\mathrm{H}$, et al. Assessment of enthesitis in ankylosing spondylitis. Ann Rheum Dis 2003;62(2):127-32.

23. Moll J y Wright V. Psoriatic Arthritis. Semin Arthritis Rheum. 1973;3(1):55-78.

24. Ritchlin CT, Kavanaugh A Gladman, DD, Mease PJ, Helliwell P, et al. Treatment recommendations for psoriatic arthritis. Ann Rheum Dis 2009;68(9):13871394.

25. INDEC, Ministerio de Salud, Presidencia de la Nación Argentina. Tercera encuesta Nacional de factores de riesgo para enfermedades no transmisibles. 10 de Julio de 2015. Buenos Aires, Argentina. ISBN: 978-950-380218-2.

26. Statistics Canada. Canadian Community Health Survey (CCHS) Cycle 3.1 (2005) Public Use Microdata File (PUMF) User Guide. Ottawa: Statistics Canada, 2006.

27. Hubert HB, Feinleib M, Macnamara PM, Castelli WP. Obesity as an independent risk factor for cardiovascular disease: a 26-year follow-up of participants in the Framingham Heart Study. Circulation 1983;67(5):968977.

28. Neogi T, Zhang Y. Epidemiology of osteoarthritis. Rheum Dis Clin North Am 2013;39(1):1-19.
29. Sandell LJ. Obesity and osteoarthritis: is leptin the link? Arthritis Rheum 2009;60(10):2858-2860.

30. Di Minno MN, Peluso R, Iervolino S, Lupoli R, Russolillo A, Scarpa R, et al. Obesity and the prediction of minimal disease activity: a prospective study in psoriatic arthritis. Arthritis Care Res (Hoboken) 2013;65(1):141-7.

31. Di Minno MN, Peluso R, Iervolino S, Russolillo A, Lupoli R, Scarpa R, et al. Weight loss and achievement of minimal disease activity in patients with psoriatic arthritis starting treatment with tumour necrosis factor alpha blockers. Ann Rheum Dis 2014;73(6):1157-62.

32. Wang Y, Chen J, Zhao Y, Geng L, Song F, Chen HD. Psoriasis is associated with increased levels of serum leptin. Br J Dermatol 2008;158(5):1134-5.

33. Takahashi H, Tsuji H, Takahashi I, Hashimoto Y, Ishida-Yamamoto A, Iizuka H. Plasma adiponectin and leptin levels in Japanese patients with psoriasis. $\mathrm{Br}$ J Dermatol 2008;159(5):1207-8.

34. Eder L, Jayakar J, Pollock R, Pellett F, Thavaneswaran A, Chandran V, et al. Serum adipokines in patients with psoriatic arthritis and psoriasis alone and their correlation with disease activity. Ann Rheum Dis 2013;72(12):1956-61. 